\title{
Stereotactic Body Radiation Therapy for Liver Metastases: Single Institution Experience
}

\author{
Rute Pocinho ${ }^{1}$, David Roberge ${ }^{2}$ \\ 1. Department of Radiotherapy - Instituto Português de Oncologia de Lisboa Francisco Gentil 2. \\ Department of Oncology, Division of Radiation Oncology, McGill University Health Center
}

$\square$ Corresponding author: Rute Pocinho, selenis@gmail.com

Disclosures can be found in Additional Information at the end of the article

\section{Abstract}

Background: Stereotactic body radiation therapy (SBRT) is an emerging local treatment for limited liver metastases. We report a single institution experience with liver metastases treated with SBRT.

Methods: Twenty-three consecutive patients treated with SBRT for 27 liver metastases: eight women and 15 men, median age 69 years (26 to 87). SBRT was the chosen therapy when the disease was considered life-limiting and unsuitable for resection or radio-frequency ablation. The median radiation dose was $40 \mathrm{~Gy}$ (20 to $50 \mathrm{~Gy}$ ) delivered in one to 10 fractions. Response to treatment was measured according to RECIST criteria on post-treatment CT, MRI and/or PET imaging. Acute and late toxicities were graded according to CTCAE v4.0.

Results: Twenty-two patients completed SBRT. One stopped treatment after three fractions due to biliary obstruction from progressive tumor. Treatment was well-tolerated, with three patients (13.6\%) presenting with Grade 1 and two (9\%) presenting with Grade 2 acute gastrointestinal toxicity. Median follow-up was 15 months (range: 3-43) with one patient lost to follow-up. A single patient had symptomatic colitis that resolved with conservative treatment. Complete response was initially achieved in eight of 25 (32\%) lesions, partial response in four (16\%), disease stabilization in 12 (48\%), and continued progression in one (4\%). At last follow-up, six of 21 patients (28.6\%) had progression of a treated lesion. Actuarial overall one-year and two-year survival rates were $94 \%$ and $60 \%$, respectively (median 30.1 months). Actuarial one and two-year local control rates were $62 \%$.

Conclusions: SBRT is a promising well-tolerated treatment for non-resectable liver metastases.

Published 05/01/2012

(c) Copyright 2012

Pocinho et al. This is an open access article distributed under the terms of the Creative Commons Attribution License CC-BY 3.0., which permits unrestricted use, distribution, and reproduction in any medium, provided the original author and source are credited.
Categories: Internal Medicine, Radiation Oncology

Keywords: liver metastases, stereotactic body radiation therapy, sbrt

\section{Introduction}

The liver is a common site of metastatic disease. Hepatic involvement can lead to serious morbidity and is often life-limiting. Approximately half of metastatic death from breast and prostate cancers are associated with liver metastases. Death due to colorectal cancer is commonly related to liver metastases, often the only site of metastatic disease.

When liver involvement is limited, aggressive local treatment is a therapeutic option that can 
lead to long-term disease control [1-4].

For many primary tumors sites, systemic therapy may allow for transitory responses and increased median survival. Infrequently utilized, palliative whole liver radiotherapy may offer some symptomatic relief [5]. Surgical resection of hepatic metastasis, typically for colorectal cancers, can lead to five year overall survival rates of up to 58\% [6]. Nonetheless, clinical and technical limitations narrow eligibility to metastasectomy. Limitations may relate to: intrahepatic location of the metastases, surgical plans which would not spare sufficient healthy liver, medical co-morbidities, and patient refusal. Only about 10 to $25 \%$ of patients with liver metastases from colorectal cancer can benefit from curative resection [7-8].

Nowadays, new therapeutic options have been developed for the remaining patients, enabling radical treatment without or in combination with surgery: microwave ablation, radiofrequency ablation (RFA), and stereotactic body radiation therapy (SBRT).

RFA is limited to lesions measuring less than $4-5 \mathrm{~cm}$. Radiofrequency is generally avoided for lesions adjacent to large vessels and those sub-capsular locations. Treatment of anatomically unfavourable lesions could lead to a tumor-sparing heat sink effect and/or a risk of thermal injury to vessels, biliary structures or adjacent organs. Major complications are seen in about $5 \%$ of the patients after RFA: portal thrombosis, hemoperitoneum, colic perforation, seeding of the catheter trajectory or hepatic abscess.

Radiotherapy for liver metastasis was originally limited by radiation-induced liver disease (RILD), which occurred with therapeutic doses of whole-liver irradiation. Liver tolerance to radiotherapy depends on treatment volume and fractionation. In standard fractionation, severe RILD occurs with increasing frequency at whole-liver doses of $\geqslant 36 \mathrm{~Gy}$, and is much rarer with mean doses inferior to $31 \mathrm{~Gy}$. An analysis of over 200 patients with hepatic tumors treated at University of Michigan demonstrated that much higher doses of radiation can be delivered to a small volume of liver. For irradiated volumes below $25 \%$ of the liver, a high dose (>100 Gy in standard fractions) can be given with a low risk of RILD [9]. Patients with liver metastases are less prone to have underlying liver disease as is often the case of patients with primary liver tumors. They are thus more tolerant of liver-directed therapies.

SBRT allows delivery of high doses per fraction to small volumes with geographic sparing of adjacent organs at risk. Published series of SBRT for liver metastases report median survival time of six months to 4.3 years, with actuarial local control at one to two years, ranging from 67 to $92 \%[10-14]$.

In the present review, we report a single institution's experience with liver metastases treated with SBRT.

\section{Materials And Methods}

\section{Patients}

A multidisciplinary tumor board composed of surgeons, radiation oncologists and medical oncologists reviewed cases and made recommendation for systemic and liver-directed therapies. Stereotactic body radiotherapy was the preferred treatment when the disease was considered life-limiting and unsuitable for resection or radio-frequency ablation.

Patients were considered for SBRT when they had a limited number of lesions ( $\leqslant 3$ lesions) and 700 cc of liver could be spared. Extrahepatic disease did not per se exclude patients from SBRT. Through review of the radiation-oncology SBRT database, 23 consecutive patients were 


\section{Cureus}

identified. These patients were treated with SBRT for 27 liver metastases from November 2006 to September 2010. Clinical information was collected retrospectively from the electronic charts of the patients at McGill University. All patients gave informed consent for their treatment.

Patients had a median age of 69 (range: 26 to 87) at the time of SBRT. There were eight women and 15 men.

Nine (39\%) patients had extra-hepatic metastatic disease, and 14 (61\%) had been previously treated with chemotherapy. One to three lesions (median one lesion) were treated in each patient. Table 1 shows patient characteristics.

\section{Characteristic}

Age, years

Sex F:M

Primary site

Colon

Rectum

Breast

Gastric

Nasopharynx

Pancreas

Bladder

Leiomiosarcoma

Prostate

Endometrium

Ovary

Number of Lesions

3

Lesion Dimension

Dose, Gy

Dose per fraction, Gy

Number of fractions
Patients, $\mathrm{n}=\mathbf{2 3}$

Median 69 (26 - 87)

8:15

$12(52.2 \%)$

$2(8.7 \%)$

$1(4.3 \%)$

$1(4.3 \%)$

$1(4.3 \%)$

$1(4.3 \%)$

$1(4.3 \%)$

$1(4.3 \%)$

$1(4.3 \%)$

$1(4.3 \%)$

$1(4.3 \%)$

$21(91,3 \%)$

$2(8.7 \%)$

Mean 3.2cm (0.7 - 10.6

Median 40 Gy (20 - 50)

Median 6 Gy (3 - 20)

$1(4.3 \%)$ 


\section{Cureus}

3

4

5

6

10

Previous chemotherapy

Extra-hepatic metastases
$6(26 \%)$

$2(8.7 \%)$

$1(4.3 \%)$

$2(8.7 \%)$

$11(47.8 \%)$

$14(61 \%)$

$9(39 \%)$

\section{TABLE 1: Patient Characteristics}

\section{Radiation treatments}

Twenty patients (24 lesions) received SBRT from a conventional linear accelerator (Linac), and three (three lesions) were treated with the CyberKnife radiosurgery system (Accuray, Sunnyvale, CA).

Fiducials for daily image-guided radiotherapy (IGRT) were implanted percutaneously in peripheral intratumoral locations with computed tomography (CT) (22 patients) or ultrasound (one patient) guidance. One to four fiducials were placed in each liver [15].

Patients were either immobilised with a double-vacuum cushion system or a simple vacuum cushion. Three and four dimensional simulation images were co-registered, when possible, to diagnostic MRI or CT images. Gross tumor volume (GTV) was expanded by 0-5 $\mathrm{mm}$ to for a clinical target volume (CTV). An additional 3-7 mm were added to produce planning target volume (PTV). Four to 10 fields were used for isocentric Linac planning, whereas 59 to 129 beams were used for in CyberKnife cases. The median radiation dose was $40 \mathrm{~Gy}$ (20 to $50 \mathrm{~Gy}$ ) delivered in one to 10 fractions. Seven (30\%) patients were enrolled in a multi-institutional clinical trial. Treatments were delivered with respiratory gating, so-called adaptive gating (ExacTrac, BrainLab, Feldkirchen, Germany) or predictive tumor tracking.

Patients were monitored for in-field and out-of-field local recurrences, as well as extra-hepatic recurrences with CT, magnetic resonance imaging (MRI), and/or positron-emission tomography (PET). Overall survival was also calculated on an actuarial basis.

Initial response to treatment was measured according to Response Evaluation Criteria in Solid Tumors (RECIST) criteria three to six months after SBRT. Local progression was defined as an increase of at least $20 \%$ in a treated lesion. Time to local progression was measured from the first day of SBRT. For survival, patients were censored at the time of last contact.

Acute and late toxicities were graded according to National Cancer Institute (NCI) Common Terminology Criteria for Adverse Events (CTCAE) version 4.0. Acute toxicity was defined as events occurring within the first three months after SBRT.

\section{Statistics}

GraphPad Prism ${ }^{\circledR}$ version 5.0 for Windows (GraphPad Software, San Diego, CA) was used to 
calculate and chart actuarial survival and local control results.

\section{Results}

Out of the 23 initial patients, 22 completed the SBRT treatment, making for 26 lesions, measuring 0.7 to $10.6 \mathrm{~cm}$ (median $3.2 \mathrm{~cm}$ ). One patient with gastric cancer stopped treatment after three fractions due to biliary obstruction from progressive tumor. Fourteen patients (63.6\%), with 16 lesions had colorectal cancer.

Median GTV volume was $10.65 \mathrm{~mL}$ (0.6 to $312.5 \mathrm{~mL}$ ), and median contoured liver volume was $1497 \mathrm{~mL}$ (938 to $3400 \mathrm{~mL}$ ).

Treatment was well-tolerated, with no RILD or serious liver toxicity observed. Three (13.6\%) patients had Grade 1 acute toxicity (right upper quadrant discomfort in three patients) and two (9\%) had Grade 2 toxicity - one with diarrhoea and another with both nausea and vomiting. As for late toxicity, a single patient had symptomatic colitis (Grade 2), which resolved with conservative treatment.

With one patient lost to follow-up, 21 patients with 25 lesions were followed for a median of 15 months (range: 3-43 months).

Complete response was initially achieved in eight of 25 (32\%) lesions, partial response in four (16\%), disease stabilization in 12 (48\%), and continued progression in one (4\%).

At last follow-up, six of 21 patients (28.6\%) had progression of a treated lesion, and the one and two year local control rate was $62 \%$ (Figure 1 ).

Actuarial overall one year and two year survival rates were $94 \%$ and $60 \%$, respectively (Figure 1) (median 30.1 months). Overall survival was not significantly different between patients with colorectal vs. other primary malignancies.
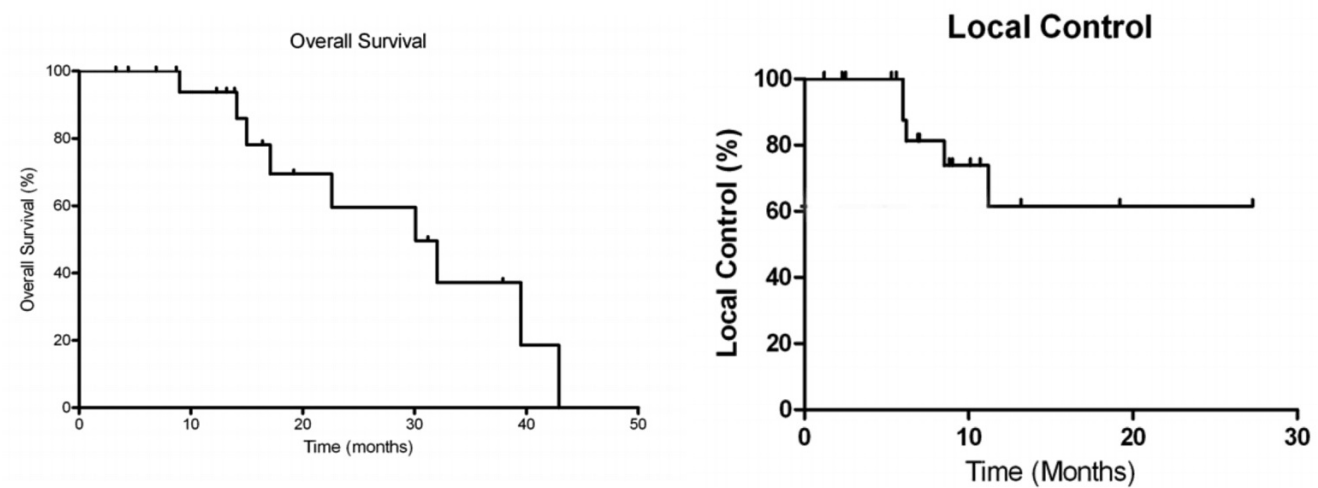

\section{FIGURE 1: Overall Survival and Local Control}

The graphics illustrate actuarial overall survival and local control for our study population.

\section{Discussion}

This retrospective study corroborates the safety of SBRT in the treatment of liver metastases not suitable for surgery or radiofrequency ablation. As with other SBRT studies [14, 16-17], treatment was generally well-tolerated, with mild acute toxicity and a low Grade 2 or higher 
late toxicity incidence in this series. Despite treatment of large lesions, only a single patient (4.5\%) developed significant late toxicity in the form of symptomatic colitis which resolved with conservative treatment. Reduced treatment volumes through the use of fiducials, tracking, and adaptative gating may have contributed to reducing toxicity. As the dose-limiting organ(s) vary from case to case, the number of patients treated is still insufficient to fully describe the tolerance of the adjacent organs to high dose delivered to small volumes. Likewise, liver tolerance using hypofractionation is not well understood. Given the low symptomatic hepatotoxicity seen in our study and in the literature, there might be room for escalation in normal tissue tolerance levels [17].

In the last few years, advances in systemic therapy have resulted in a better prognostic for patients with metastatic colorectal cancer, with median survivals reaching two years [18]. For the patients with limited liver metastatic disease amenable to surgical resection, five year survival can reach up to $43 \%$ [19-21], thus demonstrating the importance of an aggressive local treatment approach. In complement to surgery, microwave ablation, and RFA, SBRT is becoming an increasingly used local therapy, applying the principles of radiosurgery, widely used for brain tumors, to tumors outside the brain.

Different liver SBRT studies report local control rates ranging from 55 to $97 \%$ at one to to years [9, 13-14, 16-17, 22]. This wide range can be due to patient, treatment and follow-up heterogeneity across studies.

In our study, with a wide range of histologies and fractionation, a local control rate of $62 \%$ at two years was calculated. It is within but on the lower side of the range of reported series. This may be explained by the varied histologies and the different tumor number and sizes treated (some of the lesions were larger than typical in most studies). Another factor may be the total dose, lower than in some other reports - median radiation dose was $40 \mathrm{~Gy}$ (20 to $50 \mathrm{~Gy}$ ) - and dose per fraction. Several of the patients were treated in a Phase I trial which used conservative dosing schemes as a starting point for escalation. Chang, et al. demonstrated that local control for colorectal metastases is dose-dependent, with 18-month local control diminishing from 84 to $43 \%$ when total dose was under 42 Gy [17].

The impact of SBRT on survival is not yet determined [17]. Upcoming years may see prospective comparisons to other local modalities and evaluation of SBRT in the treatment of oligometastatic disease in cancers where this is not yet a widespread standard (small cell lung, for example). Many factors will influence overall survival, including active extra-hepatic disease. Extra-hepatic disease has shown to correlate with worse survival in other surgical [18, 23] and SBRT [17] series. Actuarial survival at one to two years with SBRT for liver metastases has been reported in the literature to range from $71-94 \%$ and $30-62 \%$, respectively [24].

In our population, with $39 \%$ of the patients having active extra-hepatic disease, actuarial one and two year survival rates were 94 and 60\%, respectively, which is in agreement with the published literature. Our patient numbers are too small to reliably tease out prognostic factors.

\section{Conclusions}

Our series contributes to a growing literature indicating that SBRT is a feasible, safe and effective treatment for selected liver metastases.

\section{Additional Information \\ Disclosures}

Human subjects: All authors have confirmed that this study did not involve human 
participants or tissue. Animal subjects: All authors have confirmed that this study did not involve animal subjects or tissue. Conflicts of interest: In compliance with the ICMJE uniform disclosure form, all authors declare the following: Payment/services info: All authors have declared that no financial support was received from any organization for the submitted work. Financial relationships: All authors have declared that they have no financial relationships at present or within the previous three years with any organizations that might have an interest in the submitted work. Other relationships: All authors have declared that there are no other relationships or activities that could appear to have influenced the submitted work.

\section{References}

1. Kemeny MM, Adak S, Gray B, et al.: Combined- modality treatment for resectable metastatic colo rectal carcinoma to the liver: Surgical resection of hepatic metastases in combination with continuous infusion of chemotherapy-An intergroup study. J Clin Oncol. 2002, 20:14991505.

2. Harrison LE, Brennan MF, Newman E, et al.: Hepatic resection for noncolorectal, nonneuroendo crine metastases: A fifteen-year experience with ninety-six patients. Surgery. 1997, 121:625-632.

3. Wei AC, Greig PD, Grant D, et al.: Survival after hepatic resection for colorectal metastases: A 10-year experience. Ann Surg Oncol. 2006, 13:668-676.

4. Ercolani G, Grazi G, Ravaioli M, et al.: The role of liver resections for noncolorectal, nonneuroendo crine metastases: Experience with 142 observed cases. Ann Surg Oncol. 2005, 12:459-466.

5. Bydder S, Spry NA, Christie DR, et al.: A prospective trial of short-fractionation radiotherapy for the palliation of liver metastases. Australas Radiol. 2003, 47:284-288.

6. Abdalla EK, Vauthey JN, Ellis LM, et al.: Recurrence and outcomes following hepatic resection, radiofrequency ablation, and combined resection/ablation for colorectal liver metastases. Ann Surg. 2004, 239:818-825.

7. Nordlinger B, Quilichini MA, Parc R, et al.: Hepatic resection for colorectal liver metastases. Influence on survival of preoperative factors and surgery for recurrences in 80 patients. Ann Surg. 1987, 205:256-263.

8. De Bari B, Guillet M, Mornex F: Radiothérapie en conditions stéréotaxiques des métastases hépatiques. Cancer Radiother. 2011, 15:72-76. 10.1016/j.canrad.2010.11.005

9. Dawson LA, Normolle D, Balter JM, et al.: Analysis of radiation-induced liver disease using the Lyman NTCP model. Int J Radiat Oncol Biol Phys. 2002, 53:810-821.

10. Herfarth KK, Debus J, Lohr F, et al.: Stereotactic single-dose radiation therapy of liver tumors: results of a phase I/II trial. J Clin Oncol. 2001, 19:164-170.

11. Milano MT, Katz AW, Muhs AG, et al.: A prospective pilot study of curative-intent stereotactic body radiation therapy in patients with 5 or fewer oligometastatic lesions. Cancer. 2008, 11:650-658.

12. Méndez Romero A, Wunderink W, Hussain SM, et al.: Stereotactic body radiation therapy for primary and metastatic liver tumors: a single institution phase i-ii study. Acta Oncol. 2006, 45:831-837.

13. Wulf J, Guckenberger M, Haedinger U, et al.: Stereotactic radiotherapy of primary liver cancer and hepatic metastases. Acta Oncol. 2006, 45:838-847.

14. Lee MT, Kim JJ, Dinniwell R, et al.: Phase I study of individualized stereotactic body radiotherapy of liver metastases. J Clin Oncol. 2009, 27:1585-1591. 10.1200/JCO.2008.20.0600

15. Roberge D, Cabrera T: Percutaneous liver fiducial implants: techniques, materials and complications. Liver Biopsy in Modern Medicine. Yoshiaki Mizuguchi (ed): InTech, Rijeka, Croatia; 2011. 107-116. 10.5772/21170

16. Kavanagh BD, Schefter TE, Cardenes HR, et al.: Interim analysis of a prospective phase I/II trial of SBRT for liver metastases. Acta Oncol. 2006, 45:848-855.

17. Chang DT, Swaminath A, Kozak M, et al.: Stereotactic body radiotherapy for colorectal liver metastases - a pooled analysis. Cancer. 2011, 117:4060-4069. 10.1002/cncr.25997

18. Fuchs CS, Marshall J, Mitchell E, et al.: Randomized, controlled trial of irinotecan plus infusional, bolus, or oral fluoropyrimidines in first-line treatment of metastatic colorectal cancer: results from the BICC-C Study. J Clin Oncol. 2007, 25:4779-4786. 


\section{Cureus}

19. Bolton JS, Fuhrman GM: Survival after resection of multiple bilobar hepatic metastases from colorectal carcinoma. Ann Surg. 2000, 231:743-751.

20. Shah SA, Bromberg R, Coates A, et al.: Survival after liver resection for metastatic colorectal carcinoma in a large population. J Am Coll Surg. 2007, 205:676-683.

21. Fong Y, Cohen AM, Fortner JG, et al.: Liver resection for colorectal metastases. J Clin Oncol. 1997, 15:938-946.

22. Hoyer M, Roed H, Traberg Hansen A, et al.: Phase II study on stereotactic body radiotherapy of colorectal metastases. Acta Oncol. 2006, 45:8223-830.

23. Carpizo DR, D’Angelica M: Liver resection for metastatic colorectal cancer in the presence of extrahepatic disease. Lancet Oncol. 2009, 10:801-809. 10.1016/S1470-2045(09)70081-6

24. Vautravers-Dewas C, Dewas S, Bonodeau F, et al.: Image-guided Robotic Stereotactic Body Radiation Therapy for Liver Metastases: is there a Dose-Response Relationship?. Int J Radiat Oncol Biol Phys. 2011, 81:39-47. 10.1016/j.ijrobp.2010.12.047 